\title{
Estudo da rede de colaboração científica em nanotecnologia na Empresa Brasileira de Pesquisa Agropecuária
}

\author{
Study of the scientific collaboration network in nanotechnology at \\ the Brazilian Agricultural Research Corporation
}

\author{
Marcelo Moreira CAMPOS'1 \\ Marcos Vinícius Amorim Ferreira GUIMARÃES² \\ Liliane Juvência Azevedo FERREIRA ${ }^{3}$ \\ Lillian Maria Araújo de Rezende ALVARES ${ }^{4}$
}

\section{Resumo}

O estudo da colaboração científica permite observar resultados que podem orientar a gestão da pesquisa em uma organização. Nesse sentido, o objetivo deste estudo é analisar a rede de colaboração científica em nanotecnologia na Empresa Brasileira de Pesquisa Agropecuária e sua evolução, considerando-se ser um tema que vem gerando resultados inovadores para o agronegócio brasileiro. Para tanto, foi empregada a metodologia de Análise de Redes Sociais, com o objetivo de perceber as relações de coautoria e de como analisar a evolução dessa rede de colaboração. Para trabalhar a perspectiva dos dados, foi escolhida a Base de Dados da Pesquisa Agropecuária, de acesso público, organizada e gerenciada pela Embrapa. A análise dos dados baseou-se na produção científica entre 2006 e 2014. Para a análise da evolução da rede, o período foi dividido em três fases: de 2006 a 2008, de 2009 a 2011 e de 2012 a 2014. Os dados, recuperados a partir do ano de 2006, permitiram compor um período significativo de análise de quase dez anos, evidenciando que a evolução da rede aconteceu de forma progressiva, e que a colaboração científica sobre o tema na Embrapa se torna cada vez mais efetiva e dinâmica.

Palavras-chave: Análise de redes sociais. Colaboração científica. Embrapa. Nanotecnologia.

\footnotetext{
Abstract

The study of scientific collaboration allows us to observe, for instance, results that can guide research management in an organization. In this sense, the objective of this study is to analyze the network for scientific collaboration in nanotechnology at the Brazilian Agricultural Research Corporation, and the evolution thereof, since this is a field which has produced innovative results for Brazilian agribusiness. To this end, the methodology of Social Network Analysis was used in order to identify co-authorship connections and to analyze the evolution of this network. The origin of the data was Agricultural Research Database (Base de Dados da Pesquisa Agropecuária), which is freely accessible to the public. The analysis was based on scientific production from 2006 to 2014. For the analysis of network evolution, this timeframe was divided into three periods, consisting of the periods from 2006 to 2008, 2009 to 2011, and 2012 to 2014. The results showed that the data represents a significant timeframe for analysis, nearly 10 years, providing the perception that, insofar as the evolution of the network is concerned, it evidently occurred in a gradual manner, highlighting the fact that scientific collaboration in nanotechnology at Embrapa becomes increasingly effective and dynamic.

Keywords: Social network analysis. Scientific collaboration. Embrapa. Nanotechnology.

1 Empresa Brasileira de Pesquisa Agropecuária, Departamento de Pesquisa e Desenvolvimento. Parque Estação Biológica, PqEB W3 Norte (final), Asa Norte, 70770-901, Brasília, DF, Brasil. Correspondência para/Correspondence to: M.M.CAMPOS.E-mail:<marcelo.campos@embrapa.br>

2 Ministério da Ciência, Tecnologia e Inovação, Secretaria de Política de Informática. Brasília, DF, Brasil.

3 Universidade Federal de Goiás, Biblioteca Central, Sessão de Referência. Goiana, GO, Brasil.

4 Universidade de Brasília, Faculdade de Ciência da Informação, Programa de Pós-Graduação em Ciência da Informação. Brasília, DF, Brasil. Recebido em 19/10/2015, reapresentado em 5/2/2016 e aceito para publicação em 14/3/2016
} 


\section{Introdução}

Estudar a colaboração científica de uma área de conhecimento específica permite, por exemplo, identificar formas de transferência de novos conhecimentos, especialmente do conhecimento tácito, e também observar resultados que podem orientar a gestão da pesquisa em uma organização. Além do mais, explicam Katz e Martin (1997), o trabalho de colaboração científica não requer somente o conhecimento técnico e científico, mas também habilidades gerenciais e sociais para o trabalho colaborativo.

O avanço da Ciência acontece, em grande parte, pela interação entre os cientistas. "A colaboração científica tem sido definida como dois ou mais cientistas trabalhando juntos em um projeto de pesquisa, compartilhando recursos intelectuais, econômicos e/ou físicos" (VANZ; STUMP, 2010, p.44). Digiampietri et al. (2014) observam que talvez mais importante que o financiamento da ciência em centros, ainda em consolidação, é inseri-los efetivamente nas redes acadêmicas. Ou seja, a atuação dos cientistas em conjunto torna-se fundamental para a evolução de temas científicos.

Ziman (1979) explica que as ligações intelectuais entre as ideias dos cientistas se estabelecem por meio de relações sociais. Silva (2002) confirma essa ideia quando afirma que a sociedade contemporânea encontra-se em uma imensa rede e a produção da Ciência ocorre coletivamente, a partir da ligação de várias competências e da associação dos níveis sociais, técnicos e financeiros. Assim, a análise de redes constitui "o meio para realizar uma análise estrutural cujo objetivo é mostrar em que a forma da rede é explicativa dos fenômenos analisados" (MARTELETO, 2001, p.72).

Tema emergente na Ciência, a Nanotecnologia tem aplicabilidade com grande potencial transformador. Para este estudo, a escolha do tema deu-se em razão de ser uma área estratégica de pesquisa para o agronegócio brasileiro, envolvendo estudos multidisciplinares e contribuindo para o desenvolvimento econômico e social do país (ALCÁZAR et al., 2011). Estudos acerca do tema, conforme observam Alcázar et al. (2011), vêm gerando redes de pesquisa ao redor do mundo e fortalecendo as redes de inovação, caso em que se inclui a participação da Empresa Brasileira de Pesquisa Agropecuária (Embrapa).

A Empresa Brasileira de Pesquisa Agropecuária, criada em 1973, é vinculada ao Ministério da Agricultura, Pecuária e Abastecimento. Tem como missão "viabilizar soluções de Pesquisa, Desenvolvimento e Inovação para a sustentabilidade da agricultura, em benefício da sociedade brasileira" (EMPRESA BRASILEIRA DE PESQUISA AGROPECUÁRIA, 2008, p.18). Ao longo de sua existência, possibilitou a consolidação do Brasil como líder em pesquisas sobre agricultura tropical, além da consolidação de um "ciclo virtuoso de pesquisa e inovação da agropecuária brasileira" (EMPRESA BRASILEIRA DE PESQUISA AGROPECUÁRIA, 2014a, p.6). É uma das principais responsáveis pelo desenvolvimento de uma agricultura baseada em Ciência (EMPRESA BRASILEIRA DE PESQUISA E AGROPECUÁRIA, 2014b). Atua nacionalmente por meio de 41 centros de pesquisa, 5 centros de serviço e 15 unidades administrativas centrais, além da diretoria executiva e do conselho de administração (EMPRESA BRASILEIRA DE PESQUISA E AGROPECUÁRIA, 2015a). Em âmbito internacional possui laboratórios de pesquisa, denominados Laboratórios no Exterior (Labex), na América do Norte, Europa e Ásia, bem como projetos na África, na América Central e na América do Sul.

Assim, este estudo visa a analisar a rede de colaboração científica em nanotecnologia na Embrapa, e sua evolução. Para tanto, foi empregada a metodologia de Análise de Rede Sociais, com o objetivo de perceber as relações de coautoria, bem como estudar a evolução dessa rede de colaboração.

\section{Redes sociais e redes de colaboração científica}

No século XIX, o conceito de redes começa a ganhar maior reconhecimento a partir de sua utilização em questões de gestão sobre tempo e espaço na explicação de fluxos (MARTINS, 2012). Partindo-se dessa premissa, é possível afirmar que uma estrutura social composta de pessoas envolvidas com objetivos comuns denomina-se "Rede Social". As unidades podem ser consideradas pessoas, ou atores de um contexto, que captam, geram, integram, distribuem e disseminam informações (TEIXEIRA; SOUZA, 2012). Segundo Marteleto (2010), nas redes sociais as informações são interpretadas por atores que compartilham seus conhecimentos indivi- 
duais, gerando benefícios em comum. Podem ser: redes sociais primárias - interações informais do dia a dia, como relacionamentos com a família e amigos, e redes sociais secundárias - interações formais com grupos institucionalizados na defesa de objetivos comuns e compartilhamento de experiências.

Com base nessa categorização de Marteleto (2010), conclui-se que as Redes de Colaboração Científica enquadram-se como Redes Sociais Secundárias, uma vez que interligam dois ou mais cientistas que trabalham juntos, em um projeto de pesquisa, compartilhando recursos intelectuais, econômicos e suas experiências. De acordo com Newman (2001), essas redes podem formar pequenos mundos, e seus pares, escolhidos aleatoriamente, são ligados a partir de intermediários, com dois cientistas conectados em uma mesma rede de colaboração científica se tiverem, em comum, autores com os quais dividem a autoria. Para Vanz e Stump (2010), o colaborador de uma Rede Social de Colaboração Científica pode ser qualquer indivíduo que contribui diretamente para uma parte da pesquisa.

A Análise de Redes Sociais é uma metodologia interdisciplinar com origem na Antropologia e Sociologia, que visa a analisar as configurações dos relacionamentos em um sistema, utilizando-se de mapeamentos das relações entre indivíduos em um dado contexto, ilustrados por meio de matrizes, gr afos, análises quantitativas e qualitativas (WASSERMAN; FAUST, 1994).

A Análise de Redes Sociais conta com ferramentas computacionais específicas para visualização dos padrões das redes e realização do cálculo de algumas medidas que ajudam a analisar e visualizar as redes em estudo. Essas medidas são classificadas em três níveis de estudo: 1) atribuídas aos atores (grau, proximidade, intermediação, centralidade, prestígio, ponte, gatekeeper e isolado); 2) atribuídas às ligações (ligações indiretas, frequência, estabilidade, multiplexibilidade, intensidade, direção e simetria); 3) atribuídas às redes como um todo (tamanho, abrangência, componente, conectividade, densidade, centralização, simetria, transitividade e encadeamento) (MOORE, 2002, p.26). Para este estudo, optou-se pela utilização do software Gephi (2015).

O software Gephi é uma plataforma open source para a visualização e manipulação de grafos dinâmicos e hierárquicos, incluindo todos os tipos de redes e sistemas complexos. O usuário tem autonomia para modificar o visual e interagir com a estrutura das redes. É possível adicionar filtros destacando aspectos desejados, podendo exportar o resultado final em SVG, PNG ou PDF. Sua principal função é servir como método de análise de dados, elaboração de hipóteses, descoberta de padrões sociais e de comportamento e isolamento de estruturas importantes dentro de redes hierarquizadas. Também é largamente utilizada na visualização de redes de relações entre indivíduos e dos conteúdos que (re)produzem (MARQUEZ et al., 2013, p.3).

\section{Nanotecnologia}

O foco da nanotecnologia é a Ciência em níveis minúsculos. A pesquisa com a utilização de escala nanométrica, baseada na teoria dos átomos, remonta ao séculoV a.C. Um nanômetro é um milímetro dividido por um milhão. Trata-se de uma ciência de caráter transversal e multidisciplinar, com aplicabilidade em várias áreas do conhecimento, inclusive o agronegócio, com potencial de produzir de forma contínua resultados altamente inovadores para este que é considerado um dos setores de maior impacto para a economia brasileira. A nanotecnologia possui um alto potencial inovador, influenciando mudanças sociais e favorecendo o desenvolvimento tecnológico e a geração de novos conhecimentos. É considerada como uma área de estudos de caráter dinâmico e intensa em conhecimento (ALCÁZAR et al., 2011).

Em 1996 a Embrapa adquiriu seu primeiro microscópio de tunelamento e força atômica, que permite a visualização de objetos em escala nanométrica, e, desde então, vem desenvolvendo pesquisas à base de nanotecnologia, sendo pioneira nessa temática em relação ao agronegócio. Aqui vale destacar que um dos Centros de Pesquisa da Embrapa, a Embrapa Instrumentação Agropecuária, localizada em São Carlos (SP), abriga o Laboratório Nacional de Nanotecnologia para o Agronegócio (LNNA), considerado estratégico para o Brasil, ao desenvolver"desde sensores para avaliação de bebidas a nanoestruturas para liberação controlada de fertilizantes em solos"(SILVA, 2014, p.24). Ainda em relação ao LNNA, a revista The Economist, em sua edição de nove de setembro de 2010, citou o laboratório como o pri- 
meiro do mundo dedicado exclusivamente à agricultura (SILVA, 2014).

Como uma das consequências do amadurecimento e da participação nos avanços científicos e tecnológicos em nanotecnologia, em 2006 foi instalada na Embrapa a Rede de Pesquisa em Nanotecnologia aplicada ao Agronegócio (REDE AGRONANO, 2015), que, desde então, "vem se destacando por seu pioneirismo em aplicar a Nanociência na resolução dos problemas do Agronegócio - permeando desde o desenvolvimento de pesquisa básica à aplicada; produção e treinamento de forma de trabalho em Nano; e forte interlocução com um setor privado emergente altamente demandante de novas soluções" (EMPRESA BRASILEIRA DE PESQUISA E AGROPECUÁRIA, 2014b). Até o final de 2014, a Rede AgroNano contabilizava o envolvimento de 150 pesquisadores, sendo 70 da Embrapa, 14 Centros de Pesquisa da Embrapa, mais de 30 Universidades no Brasil e no exterior, além de 80 instituições parceiras em todo o país (ESTEVES; FRAGALLE, 2013; REYNOL, 2014). Do ponto de vista da colaboração científica, que pode gerar vínculos de longa duração entre os pesquisadores de uma rede, a Rede AgroNano já soma mais de 400 publicações na área (REYNOL, 2014).

\section{Procedimentos metodológicos}

Observando-se a natureza aplicada deste estudo e as abordagens de pesquisa qualitativa e quantitativa, ele fez uso da metodologia de Análise de Redes Sociais, sendo construído em duas etapas básicas:

1) Definição da rede e coleta de dados: para trabalhar a perspectiva dos dados, foi escolhida a Base de Dados da Pesquisa Agropecuária (EMPRESA BRASILEIRA DE PESQUISA E AGROPECUÁRIA, 2015b), pública, organizada e gerenciada pela Embrapa. O tema escolhido foi nanotecnologia, e a análise baseou-se na produção científica (seção "produção científica" na base de dados) gerada em forma de artigos e resumos em anais de congresso, artigos publicados em periódicos indexados e capítulo em livro técnico-científico. Não foram contemplados os tipos de documentos folders e folhetos, uma vez que se tratam de documentos informativos de teor comercial, sem finalidade científica, isto é, são considerados literatura comercial (trade literature), materiais produzidos por empresas e outras organizações, com o objetivo de promover a venda de seus produtos e serviços (DIAS; CAMPELLO, 2003).

A busca se deu pelo uso do termo nanotecnologia, em português, com e sem aspas, pois se observou que não houve diferença. Além disso, é importante ressaltar que o termo em inglês "nanotechnology" também é recuperado pela base, a partir da busca pelo termo em português.

Dessa forma, foram recuperados 638 registros no total, a partir do ano de 2006, correspondendo a 0,07\% dos documentos indexados na base. Na data da pesquisa, 30 de setembro de 2015, a base contava com 832257 documentos indexados, em 49 coleções. Para efeitos comparativos, na intenção de avaliar a evolução da colaboração científica, os registros foram salvos em três arquivos do tipo texto (extensão .txt), respectivamente nos períodos: 2006 a 2008; 2009 a 2011 e 2012 a 2014, lembrando que o início da alimentação da base com documentos em nanotecnologia se deu no ano de 2006. Sendo assim, recuperou-se, para cada período definido: 2006 a 2008: 49 registros; 2009 a 2011: 126 registros e 2012 a 2014: 463 registros. O número médio de autores por artigo, ao longo da pesquisa (2006-2014), totalizou 4,75. Para os períodos, as médias de autores por artigos são: 2006 a 2008: 4,04; 2009 a 2011: 4,61 e 2012 a 2014: 4,87 .

2) Normalização e tratamento dos dados: após o download dos registros na BDPA, os arquivos obtidos foram manipulados manualmente para que mantivessem somente a lista com os nomes dos autores de cada registro. Os demais dados dos registros foram excluídos. Cada lista de autores foi mantida em uma linha do arquivo, e os nomes dos autores foram separados por "ponto e vírgula", mesmo formato já disponibilizado no download da BDPA. Os arquivos foram inseridos, separadamente, no Open Refine, software open source, desenvolvido pelo Google, para tratamento de dados não formatados, permitindo a limpeza e transformação desses dados (OPEN REFINE, 2015). Esse software permite tratar dados em formatos distintos e consegue reconhecer padrões entre os dados a serem tratados, por meio de um conjunto de algoritmos específicos. Esses padrões podem ser, por exemplo, relacionados à existência de nomes semelhantes foneticamente, mas grafados de forma diferente nos registros. 
O software permitiu a identificação e remoção das inconsistências presentes nos dados, como nomes informados incorretamente, abreviados de forma diferente e até mesmo grafados com erros. Segundo Newman (2001), uma das maiores dificuldades na montagem de redes de coautoria reside na correta identificação dos autores. Barbastefano et al. (2015) observam, também, a importância que essa fase de tratamento e normalização possui para a pesquisa, principalmente em estudos de rede de coautoria. Se não houver o devido cuidado, os resultados e as métricas aplicadas poderão ser afetados negativamente, trazendo resultados equivocados. Isso porque alguns autores podem ter o mesmo nome, ou podem ter se identificado de forma diferente em diferentes publicações, usando abreviações distintas, por exemplo.

Depois de devidamente tratados no Open Refine, os dados foram salvos em formato Excel (extensão .xlsx) para posterior importação no Gephi. No Gephi foi instalado o plugin "Excel/csv converter to network", que possui uma funcionalidade para permitir a criação de redes de coautoria, desde que os nomes dos autores estejam em células de uma planilha Excel, separados por algum caractere específico, como "ponto-e-vírgula".
O software Gephi calcula diversas métricas que auxiliam no entendimento e análise da rede. No Quadro 1, são descritas algumas dessas métricas, identificadas como as mais relevantes para o entendimento da rede analisada.

\section{Resultados e Discussão}

O indicador utilizado neste estudo foi o de coautoria dos documentos coletados. Segundo Katz e Martin (1997), a colaboração científica pode ser estudada segundo outros indicadores, porém a coautoria é o indicador mais utilizado. Assim, obteve-se uma rede de coautoria com 893 nós (autores) e 3974 relações.

A rede inicial apresentada pelo Gephi é formada de maneira aleatória, posicionando os nós sem nenhuma lógica aparente. No entanto, para facilitar a análise, é possível utilizar alguns recursos do Gephi que alteram o posicionamento dos nós mediante uma determinada lógica e também permitem a apresentação do grafo de forma mais compreensiva. Para esta análise inicial da rede obtida, optou-se por trabalhar com a topologia (filtro) do componente gigante, que é o maior cluster ou grupo conectado entre si. Com a aplicação desse filtro, obser-

Quadro 1. Métricas do Gephi utilizadas para análise deste estudo.

\begin{tabular}{|c|c|c|c|c|c|}
\hline \multicolumn{6}{|c|}{ Visão geral da rede } \\
\hline Métrica & Descrição & $\begin{array}{c}\text { Valores } \\
\text { Período } \\
2006 / 2008\end{array}$ & $\begin{array}{c}\text { Valores } \\
\text { Período } \\
2009 / 2011 \\
\end{array}$ & $\begin{array}{l}\text { Valores } \\
\text { Período } \\
2012 / 2014\end{array}$ & $\begin{array}{l}\text { Valores } \\
\text { Período completo } \\
2006 / 2014 \\
\end{array}$ \\
\hline Grau Médio & Número médio de conexões (arestas) dos nós da rede. & 5,37 & 6,917 & 8,869 & 8,90 \\
\hline Diâmetro da Rede & $\begin{array}{l}\text { É a menor distância entre os dois nós mais distantes da } \\
\text { rede. É a representação do tamanho linear da rede. }\end{array}$ & 4 & 7 & 10 & 8 \\
\hline Densidade do Grafo & $\begin{array}{l}\text { Mostra o quanto a rede está próxima de ser completa. Um } \\
\text { grafo completo tem todas as arestas possíveis e densidade } \\
\text { igual a } 1 .\end{array}$ & 0,05 & 0,026 & 0,013 & 0,01 \\
\hline $\begin{array}{l}\text { Componentes } \\
\text { conectados }\end{array}$ & Número de componentes conectados na rede. & 16 & 7 & 13 & 20 \\
\hline $\begin{array}{l}\text { Coeficiente de } \\
\text { clusterização }\end{array}$ & $\begin{array}{l}\text { Indica como os nós estão conectados com sua vizinhança. } \\
\text { O coeficiente de clusterização da rede é a média do coefi- } \\
\text { ciente de clusterização dos nós. Pode identificar redes do } \\
\text { tipo small word (mundo pequeno). }\end{array}$ & 0,905 & 0,87 & 0,852 & 0,85 \\
\hline $\begin{array}{l}\text { Comprimento médio } \\
\text { de caminho }\end{array}$ & Distância média de grafo entre todos os pares de nós. & 2,137 & 3,254 & 3,205 & 3,065 \\
\hline
\end{tabular}

Fonte: Elaborado pelos autores (2016). Adaptado de Gephi versão 0.8.2 (2015). 
vou-se que a rede possui 810 nós (autores) com 3756 relações, representando 90,71\% da rede inicial obtida (Figura 1). Tal porcentagem justifica a análise isolada desse componente para o presente estudo.

De acordo com Newman (2001a; 2001b), na prática, as redes contêm aglomerações ou comunidades locais nas quais um número maior de pessoas, acima da média, se conhecem. Ou seja, os nós tendem a criar grupos altamente coesos e densos. Nesse ponto, vale observar que redes sociais são exemplos característicos de redes do tipo mundo pequeno (small worlds), descritas por Watts e Strogatz (1998), em que a distância média entre quaisquer dois elementos não ultrapassa um número pequeno de conexões, geralmente até 6 . 0 coeficiente de clusterização, descrito por Barabási (2002, p.42) como a probabilidade de que dois nós relacionados a um terceiro estejam também relacionados entre si, é um dos atributos que definem uma rede do tipo small worlds e mede o agrupamento da rede (ZHAO; GUAN, 2011). Assim, a análise da rede gerada neste estudo permite perceber que esta possui características de uma rede do tipo small worlds, pois os autores podem alcançar ou serem alcançados por outros, utilizando para tanto poucas conexões, conforme valor da métrica 'comprimento médio de caminho' da Figura 1.

\section{Evolução da Rede}

No primeiro período analisado (Figura 2), foram identificados 108 autores e 290 arestas (relações). As métricas desse período, conforme a Figura 1, apontam uma rede fragmentada, com 16 componentes isoladamente conectados, ou seja, 16 grupos com grande conectividade entre os nós, mas sem conexão entre si.

No entanto, com a aplicação da topologia do componente gigante para esse período, obtém-se uma quantidade de 42 autores e 162 relações, representando $38,89 \%$ da rede analisada no período. Ou seja, esses dados corroboram a existência de uma rede fragmentada nos três primeiros anos, talvez pela especificidade dos subtemas trabalhados dentro do tema nanotecnologia, o que pode justificar uma interação incipiente entre os autores nesse período. Essa porcentagem (38,89\%) indica também que uma análise isolada da rede no período em questão, com a aplicação do componente gigante, não representaria um grupo ideal de análise.

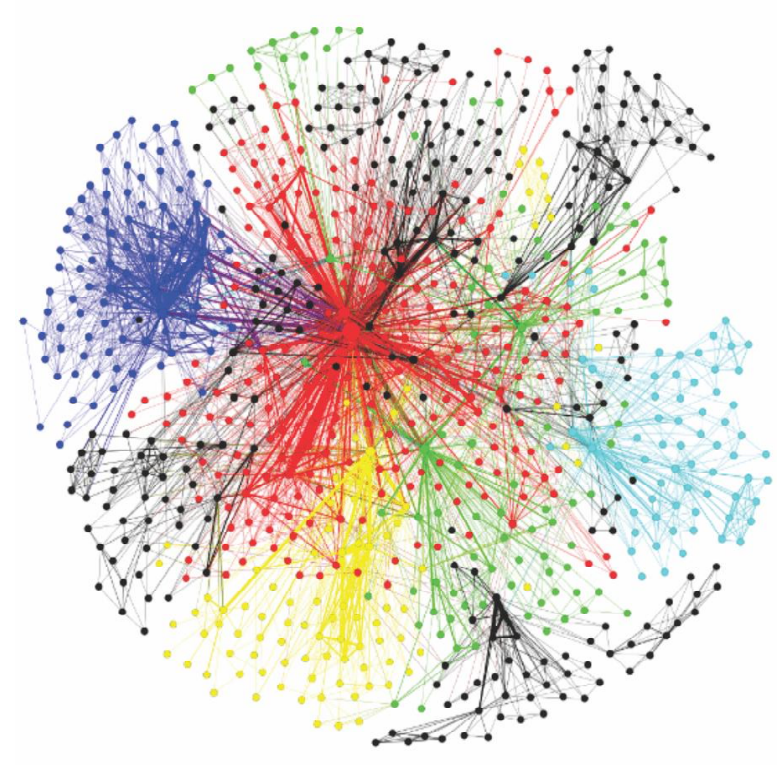

Figura 1. Representação gráfica do componente gigante da rede, no período de 2006 a 2014.

Fonte: Elaborada pelos autores (2015).

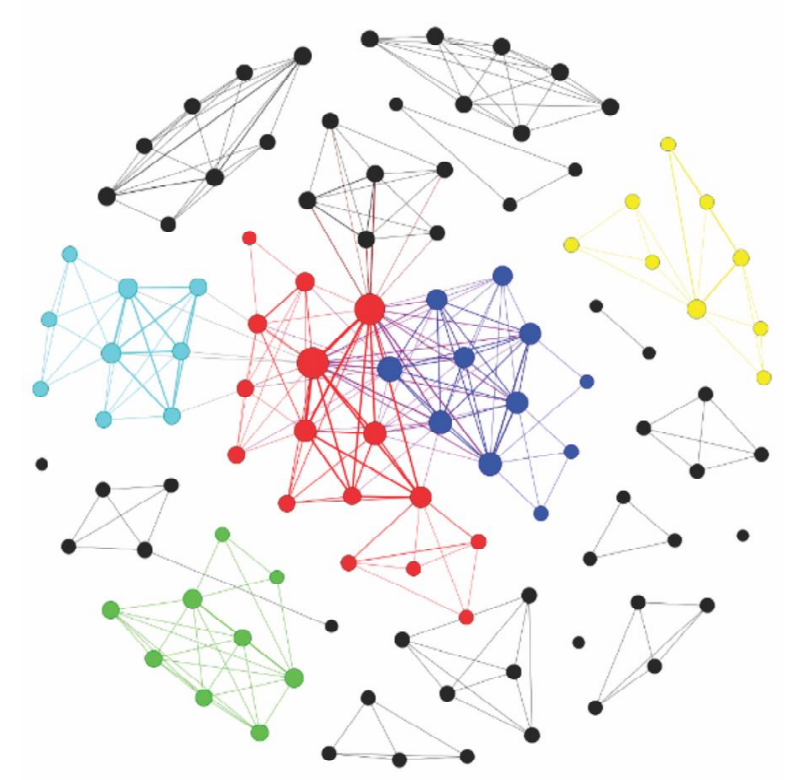

Figura 2. Rede Nanotecnologia Base de Dados da Pesquisa Agropecuária 2006/2008.

Fonte: Elaborada pelos autores (2015).

Um fato interessante em relação à recuperação dos dados a partir de 2006, refere-se à estruturação e consolidação da Rede AgroNano no início da década de 
2000, quando começaram a surgir alguns resultados das pesquisas sobre o tema na Embrapa, além da intensificação da cooperação técnica e científica com pesquisadores de instituições estrangeiras (ESTEVES; FRAGALLE, 2013).

Para o segundo período de análise, o grafo gerado apresentou 266 autores e 920 relações, percebendo-se um crescimento visível da rede em relação ao período anterior. Conforme a Figura 1, o número de componentes conectados (7) é menor que do período anterior, o que confirma uma maior conexão entre os nós. Um fato que pode ter contribuído para esse aumento, e que justifica essa análise, foi a abertura de 13 vagas para pesquisadores em nanotecnologia na Embrapa em 2006 (REYNOL, 2014 , p.14). Considerando-se o período entre lançamento do edital, realização das provas, trâmites legais de um concurso público e nomeação dos aprovados, infere-se que esse fato refletiu no aumento do número de colaboradores no período de 2009 a 2011.

Com a aplicação da topologia do componente gigante para o segundo período analisado, obtém-se uma quantidade de 219 autores e 793 relações, representando $82,33 \%$ da rede analisada no período (Figura 3). Diferentemente do percentual obtido no primeiro período, essa porcentagem indica a existência de uma rede menos fragmentada e também, se fosse o caso, já justificaria a análise isolada do período, em termos de coautoria

Fruto do amadurecimento das pesquisas, estudos e publicações sobre o tema na Embrapa, percebe-se um crescimento bem maior da rede no terceiro período analisado (2012 a 2014), em relação aos períodos anteriores, apresentando 677 autores e 3002 relações (Figura 4).

Mesmo assim, conforme as métricas apresentadas na Figura 1, nota-se pouca conexão entre os nós da rede, composta de 13 componentes conectados.

Percebe-se que o grafo gerado com os dados do terceiro período já se aproxima bastante do grafo geral, que abrange o período total de análise, compreendido entre 2006 e 2014 (Figura 1). Aplicando-se a topologia do componente gigante para o período, obtém-se uma quantidade de 622 autores e 2841 relações, representando $91,88 \%$ da rede analisada no período. Esse período

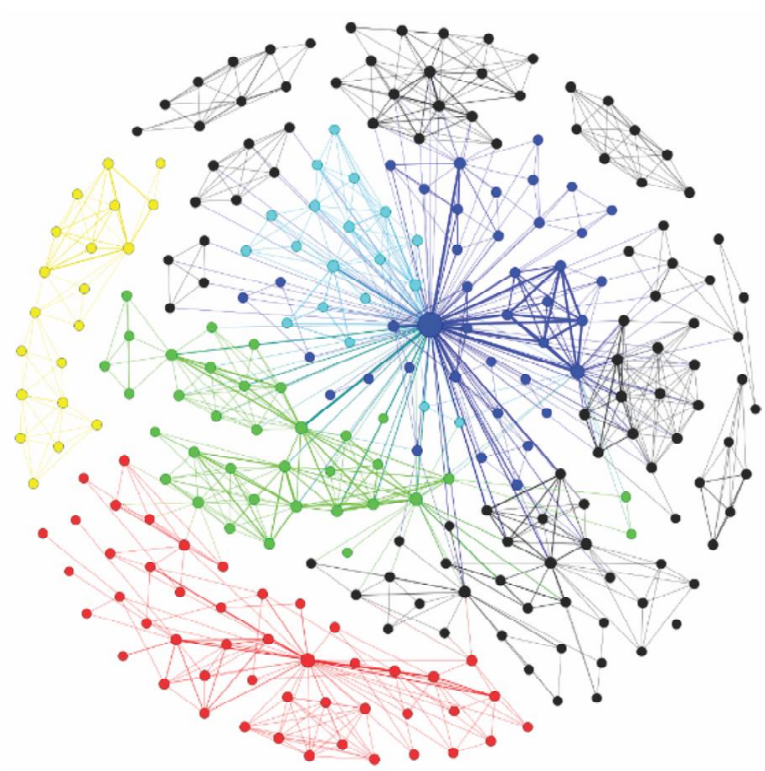

Figura 3. Rede Nanotecnologia Base de Dados da Pesquisa Agropecuária 2009/2011.

Fonte: Elaborada pelos autores (2015).

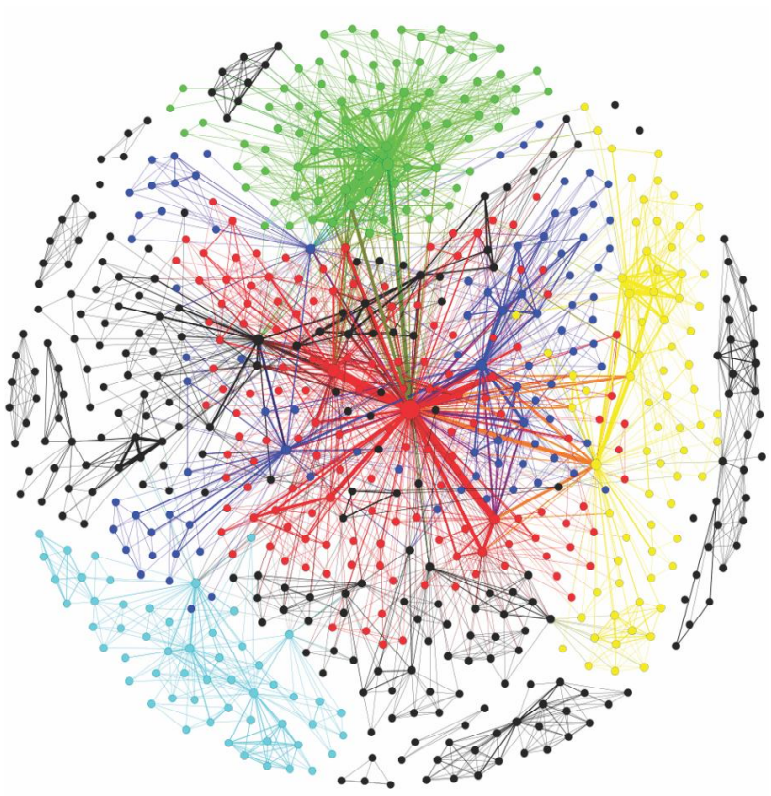

Figura 4. Rede Nanotecnologia Base de Dados da Pesquisa Agropecuária 2012/2014.

Fonte: Elaborada pelos autores (2015)

apresenta um grande crescimento da rede em número de nós e, consequentemente, em quantidade de relações. Mas é possível perceber que a rede é formada por grandes grupos conectados, que publicam intensamente 
entre seus pares, mas com pouca conexão entre esses grupos. Essa percepção pode estar relacionada à existência de Centros de Pesquisa da Embrapa geograficamente separados, o que dificultaria uma colaboração mais intensa entre pesquisadores de Centros distintos. Apesar disso, os grupos estão conectados entre si, evidenciando que a colaboração entre Centros de Pesquisa é presente, apesar de menos intensa que as colaborações intracentro.

\section{Conclusão}

Ao analisar a rede de colaboração científica em nanotecnologia na Embrapa e sua evolução, verificou-se que não se trata de uma rede fragmentada, ou seja, os pesquisadores ou grupos de pesquisadores que trabalham com o tema não o fazem de forma isolada ou sem colaboração entre si. Entretanto, notou-se que essa colaboração acontece por meio da formação de grandes grupos, em subtemas específicos, com os autores colaborando intensamente dentro do grupo e, com menos intensidade, com membros de outros grupos. Tal fato foi verificado ao se comparar o número de componentes isolados (83) com o número de autores da rede (893). Esse cenário de colaboração facilita a circulação de novos conhecimentos entre os pesquisadores e grupos da rede, de forma a estimular a inovação no tema em relação à agropecuária.

\section{Referências}

ALCÁZAR, J. J. P. et al. Avaliação de redes de inovação usando uma ferramenta baseada em redes sociais: caso brasileiro de Nanotecnologia. In: CONGRESO LATINO-IBEROAMERICANO DE GESTION TECNOLÓGICA, 14., 2011, Lima. Anais... Lima: Pontificia Universidad Católica del Perú, 2011.

BARABÁSI, A. L. Linked [Conectado]: a nova ciência dos networks: como tudo está conectado a tudo e o que isso significa para os negócios, relações sociais e ciências. São Paulo: Leopardo, 2002.

BARBASTEFANO, R. G. et al. Influência da ambiguidade de nomes na centralidade de redes de coautoria. Transinformação, v. 27, n. 3, p. 189-198, 2015. Disponível em: <http:// periodicos.puc-campinas.edu.br/seer/index.php/transinfo/ article/view/2229>. Acesso em: 21 jan. 2016.

DIAS, E.W.; CAMPELLO, B.S. Literatura comercial. In: CAMPELLO, B. S.; CENDÓN, B. V.; KREMER, J. M. (Org.). Fontes de informação parapesquisadores e profissionais. Belo Horizonte: UFMG, 2003.
Pôde-se confirmar também que se trata de uma rede não direcionada, comum em estudos de coautoria, pois essa relação (de coautoria) é assíncrona, verificando-se a formação de subgrupos específicos sobre o tema, apesar de todos estarem interconectados.

Cabe também ressaltar que, com a aplicação da topologia do componente gigante da ferramenta Gephi no terceiro período analisado (2012 a 2014), observou-se que a rede apresenta pouquíssima diferença da rede inicialmente obtida, o que leva à conclusão de que a colaboração científica em nanotecnologia, de acordo com os dados obtidos da BDPA, constitui uma rede com laços fortes.

Os dados recuperados a partir do ano de 2006 permitiram compor um período significativo de análise, de quase dez anos, o que proporcionou a percepção de que a evolução da rede ocorreu de forma crescente e positiva, evidenciando que a colaboração técnica e científica sobre o tema na Embrapa tende a se tornar cada vez mais efetiva, levando-se em consideração que se trata de um tema transversal, com aplicabilidade em áreas correlatas e em várias outras áreas do conhecimento.

\section{Colaboradores}

Todos os autores contribuíram para a concepção e desenho do estudo, análise de dados e redação final.

DIGIAMPIETRI, L. A. et al. Análise da rede de relacionamentos dos doutores brasileiros. In: CONGRESSO DA SOCIEDADE BRASILEIRA DE COMPUTAÇÃO, 34., 2014, Brasília. Anais... Brasília: Sociedade Brasileira de Computação, 2014. Disponível em: <http://csbc2014.cic.unb.br/index.php/anais-menu>. Acesso em: 22 jan. 2016.

EMPRESA BRASILEIRA DE PESQUISA AGROPECUÁRIA. Ministério da Agricultura, Pecuária e Abastecimento. Brasília: Embrapa, 2015a. Disponível em: <www.embrapa.br $>$. Acesso em: 30 set. 2015.

EMPRESA BRASILEIRA DE PESQUISA AGROPECUÁRIA. Base de Dados de Pesquisa Agropecuária. Brasília: Embrapa, 2015b. Disponível em: <http://www.bdpa.cnptia.embrapa.br>. Acesso em: 30 set. 2015.

EMPRESA BRASILEIRA DE PESQUISA AGROPECUÁRIA.V VIano Diretor da Embrapa: 2008-2011-2023. Brasília: Embrapa, 2008. 
EMPRESA BRASILEIRA DE PESQUISA AGROPECUÁRIA. O futuro do desenvolvimento tecnológico da agricultura brasileira: síntese. Brasília: Embrapa, 2014a.

EMPRESA BRASILEIRA DE PESQUISA AGROPECUÁRIA. In: WORKSHOP DA REDE DE NANOTECNOLOGIA APLICADA AO AGRONEGÓCIO, 8., 2014. Anais... São Carlos: Embrapa Instrumentação, 2014b.

ESTEVES; M.; FRAGALLE, E. Ciência globalizada. XXI: Ciência para a Vida, v. 3, p. 30-35, 2013.

GEPHI CONSORTIUM. Version 0.8.2, 2015. Paris: Médialab Sciences, 2015. Available from: <https://consortium.gephi.org/ contact.html>. Cited: Oct. 29, 2015.

KATZ, J. S.; MARTIN, B. R. What is research collaboration? Research Policy, v. 26, n. 1, p. 1-18, 1997.

MARTINS, D. L. Análise de redes sociais de colaboração científica no ambiente de uma federação de bibliotecas digitais. Tese (Doutorado em Ciência da Informação) - Escola de Comunicação e Artes, Universidade de São Paulo, São Paulo, 2012.

MARQUEZ, A. C. et al. Gephi: um software open source de manipulação e visualização de grafos. In: OFICINA GEPHI: Mapeando e analisando a vida das redes sociais. [S.I.]. Labi, 2013. Disponível em: <https://dl.dropboxusercontent.com/u/ 23392158/ApostilaOficinaGephi.pdf>. Acesso em:22 abr. 2015.

MARTELETO, R. M. Análise de redes sociais: aplicação nos estudos de transferência da informação. Ciência da Informação, v. 30, n. 1, p. 71-81, 2001.

MARTELETO, R. M. Redes sociais, mediação e apropriação de informação: situando campos, objetos e conceitos na pesquisa em Ciência da Informação. Pesquisa Brasileira em Ciência da Informação e Biblioteconomia, v. 3, n. 1, p. 27-46, 2010.

MOORE, D. L. A social netwok analysis of the national materials competency at naval air systems command. 2002. Dissertation (Master's thesis) - Naval Postgraduate School, Monterey, Califórnia, 2002.

NEWMAN, M. E. J. The structure of scientific collaboration networks. Proceedings of the National Academy os Sciences of the United States of America, v. 98, n. 2, p. 404-409, 2001 a.
NEWMAN, M. E. J. Scientific collaboration networks I: Network construction and fundamental results. Physical Review E, v. 64 n. 1, p. 1-8, 2001b.

OPEN REFINE. A free, open source, powerful tool for working with messy data. San Francisco: Google, 2015. Available from: <http://openrefine.org/community.html>. Cited: May 29, 2015.

REDE AGRONANO. Rede de Nanotecnologia Aplicada ao Agroegócio. São Carlos: Embrapa, 2015. Disponível em: <https://www.agropediabrasilis.cnptia.embrapa.br/web/ agronano-rede>. Acesso em: 30 set. 2015.

REYNOL, F. Nanotecnologia: no mundo das moléculas. Revisto XXI: Ciência para a Vida, n. 6, p. 12-24, 2014

SILVA, E. L. Rede científica e a construção do conhecimento. Informação e Sociedade: Estudos. v. 12, n. 1, p. 120-148, 2002. Disponível em:<file:///C:/Users/Cliente/Downloads/156-1531-PB.pdf>. Acesso em: 29 out. 2015.

SILVA, J. Nanotecnologia: um laboratório estratégico para o país. Revista XXI: Ciência para a Vida, n. 6, p. 24-27, 2014.

TEIXEIRA, M. R. F.; SOUZA, D. O. G. Fontes de informação em ciências: um estudo de uso a partir da metodologia de análise de redes sociais. In: SEMINÁRIO NACIONAL DE BIBLIOTECAS UNIVERSITÁRIAS, 17., 2012. Gramado. Anais... Gramado: UFRGS, 2012.

VANS, S. A. S.; STUMP, I. R. C. Colaboração científica: revisão teórico-conceitual. Perspectivas em Ciência da Informação, v. 15, n. 2, p. $42-55,2010$

ZHAO, Q:; GUAN, J. International collaboration of three 'giants' with the G7 countries in emerging nanobiopharmaceuticals. Scientometrics, v. 87, p. 159-170, 2011

ZIMAN, J. M. Conhecimento público. São Paulo: Edusp, 1979.

WASSERMAN, S.; FAUST, K. Social network analysis: Methods and applications. Cambridge: Cambridge University Press, 1994.

WATTS, D. J.; STROGATZ, S. H. Collective dynamics of "smallworld" networks. Nature, v. 393, p. 440-442, 1998. 
